\title{
AN ALGORITHM FOR SOLVING INITIAL VALUE PROBLEMS OF THIRD ORDER ORDINARY DIFFERENTIAL EQUATIONS.
}

M. O. UDO AND D. O. AWOYEMI

(Received 4, February 2009; Revision Accepted 10, June 2009)

\begin{abstract}
We propose an implicit multi-step method for the solution of initial value problems (IVPs) of third order ordinary differential equations (ODE) which does not require reducing the ODE to first order before solving. The development of the method is based on collocation of the differential system and interpolation of the approximate solution at selected grid points. This generates a system of equations, which are then solved using Gaussian elimination method. Three predictors, each of order 5, are also proposed to calculate some starting values in the main method. Analysis of basic properties is considered to guarantee the accuracy of the method. The results for method of step length $k=5$ when compared with that of step length $k=4$ show a better level of accuracy.
\end{abstract}

KEYWORD: Zero stable, third order IVPs, predictor method, step length.

\section{INTRODUCTION}

We want to consider the solution of the third order IVPs of the form.

$$
y^{l l l}=f\left(x, y, y^{l}, y^{l l}\right), y(a)=\eta_{0}, y^{l}(a)=\eta_{1}, y^{l l}(a)=\eta_{2}, a, y, f \varepsilon R-----(1.1)
$$

without reducing the problem into a system of first order IVPs. Problems of the form (1.1) are important for their application in Science and Engineering, especially in Biological Sciences and Control Theory Awoyemi (1996). Some solutions of IVPs of the form (1.1) exists. Awoyemi (1996) considered a two-step method for IVPs of order two. In his contribution Awoyemi (2003) applied the concept of P-stable method to IVPs of order three. Udo et al (2007) opined that using truncation error a linear multistep method can be derived for a second order IVP.

According to Awoyemi $(1999,2001)$, reducing to first order is inefficient due to computational burden and also uneconomical arising from computer time wastage. Bun and Vasil' Yer (1992) opined that with the reduction approach, we cannot solve equation explicitly with respect to the derivative of the highest order.

In consideration of these setbacks, we consider a method that can solve (1.1) without reduction. Eminent scholars have made efforts to solve higher order IVPs especially the special second order differential equations by a number of different methods. Lambert (1973), Enright (1974), Twizell and Khaliq (1984) independently considered the technique of multiderivative methods of solving second order IVPs. They agreed on the fact that multiderivative methods give high accuracy and possess good stability properties when used to solve first order IVPs. However, Awoyemi $(1999,2001)$ introduced the concept of multiderivative collocation approach for solving directly higher order IVPs. Awoyemi (2003) developed a P-stable method for step length $k=3$ for solving third order IVPs. Awoyemi et al (2006) considered a non-symmetric method for a step length of $\mathrm{k}=4$, also a multiderivative method for third order IVPs. Thus, in this article, we consider a step length of $k=5$ for third order IVPs instead of $k=4$ to investigate if such an extension will improve the existing result.

M. O. Udo, Department Of Mathematics And Statistics, Cross River University Of Technology, Calabar, Nigeria.

D. O. Awoyemi, Department Of Mathematical Sciences, Federal University Of Technology, Akure, Ondo State, Nigeria. 


\section{THE METHOD}

We define an operator $L$ as in Awoyemi (1999) by

$$
L=1+\sum_{j=1}^{k-2} \frac{d^{j}}{d x^{j}}
$$

where $\mathrm{k}=5$ will represent the step length of the method.

From this definition, we can rewrite equation (1.1) in the form

$$
L y=\left(1+\sum_{j=1}^{k-2} \frac{d^{j}}{d x^{j}}\right) y=f\left(x, y, y^{l}, y^{l l}\right)+\left(1+\sum_{j=1}^{k-3} \frac{d^{j}}{d x^{j}}\right) y
$$

Also let

$$
D \Psi(x)=x^{j}, j \geq 0
$$

be a canonical polynomial of degree j, Awoyemi (1999), which leads to a recurrence relation $\Psi_{j}(x)=x^{j}-\left\{j \Psi_{j-1}(x)+j(j-1) \Psi_{j-2}(x)+\ldots .+j(j-1)(j-2) \ldots(j-m+1) \Psi_{j-m}(x)\right\}, j=0(1) m--(2.4)$ Clearly, $\Psi_{j}(x)$ is a polynomial of degree $\mathrm{m}$ and it is the basis function of the proposed method.

Thus, in this work, an approximate solution of the form

$$
y(x)=\sum_{j=0}^{k} \alpha_{j} \Psi_{j}(x)
$$

is proposed. The third derivative of $(2.5)$ is

$$
y^{l l l}(x)=\sum_{j=0}^{k} j(j-1)(j-2) \alpha_{j} \Psi_{j-3}(x)
$$

where $a_{j}, j=3(1) 2 k-1$ are some of the parameters to be determined. Thus the differential system for our method is

$$
\sum_{j=0}^{k} j(j-1)(j-2) \alpha_{j} \Psi_{j-3}(x)=f\left(x, y(x), y^{l}(x), y^{l l}(x)\right)
$$

Collocating equation (2.7) at $x=x_{n+j}, j=0(1) k$ and interpolating equation (2.5) at $x=x_{n+j}, j=2(1) k-1$ yields the following system of equations

$$
\begin{aligned}
& \sum_{j=0}^{2 k-1} j(j-1)(j-2) \alpha_{i} \Psi_{j-3}\left(x_{n+j}\right)=f_{n+j}, i=3(1) 2 k-1 \\
& \sum_{j=2}^{k-1} \alpha_{i} \Psi_{j}\left(x_{n+j}\right)=y_{n+j}, i=0(1) k
\end{aligned}
$$

Solving equations (2.8),(2.9) for ' $\alpha$ ' values and substituting into equation (2.5) gives a scheme of the form

$$
y(x)=\sum_{j=2}^{k-1} \alpha_{j}(x) y_{n+j}+\sum_{j=0}^{k} \beta_{j}(x) f_{n+j}
$$

where $\alpha(x)$ and $\beta(x)$ are real coefficients of $y_{n+j}$ and $f_{n+j}$ respectively.

Noting that $f_{n+j}=f\left(x_{n+j}, y_{n+j}, y_{n+j}^{l}, y_{n+j}^{l l}\right)$, the continuous scheme of the method is given as 


$$
\begin{aligned}
& y(x)=y_{n+1}+A\left(y_{n+3}-y_{n+2}\right)+B\left(y_{n+4}-2 y_{n+3}+y_{n+2}\right)+C\left(f_{n}\right)+D\left(f_{n+1}-f_{n}\right)+ \\
& E\left(f_{n+2}-2 f_{n+1}+f_{n}\right)+F\left(f_{n+3}-3 f_{n+2}+3 f_{n+1}-f_{n}\right)+G\left(f_{n+4}-4 f_{n+3}+6 f_{n+2}-4 f_{n+1}+f_{n 2}\right) \\
& +H\left(f_{n+5}-5 f_{n+4}+10 f_{n+3}-10 f_{n+2}+5 f_{n+1}-f_{n}\right) \quad \ldots(2.11) \\
& \text { where } \\
& \mathrm{A}=\frac{1}{\mathrm{~h}}\{\mathrm{~L}+2 \mathrm{~h}) \\
& \mathrm{B} \equiv \frac{1}{2 \mathrm{~h}^{2}}\left\{\mathrm{~L}^{2}+3 \mathrm{hL}+2 \mathrm{~h}^{2}\right\} \\
& \mathrm{C}=\frac{1}{6}\left\{\mathrm{~L}^{3}+3 \mathrm{hL}^{2}+2 \mathrm{~h}^{2} \mathrm{~L}\right\} \\
& \mathrm{D}=\frac{1}{24 h}\left\{\mathrm{~L}^{4}+16 \mathrm{hL}^{3}+41 \mathrm{~h}^{2} \mathrm{~L}^{2}+26 \mathrm{~h}^{3} \mathrm{~L}\right\} \\
& \mathrm{E}=\frac{1}{240 \mathrm{~h}^{2}}\left\{2 \mathrm{~L}^{5}+35 \mathrm{hL}^{4}+240 \mathrm{~h}^{2} \mathrm{~L}^{3}+505 \mathrm{~h}^{3} \mathrm{~L}^{2}+298 \mathrm{~h}^{4} \mathrm{~L}\right\} \\
& \mathrm{F}=\frac{1}{720 \mathrm{~h}^{3}}\left\{\mathrm{~L}^{6}+18 \mathrm{hL}^{5}+130 \mathrm{~h}^{2} \mathrm{~L}^{4}+480 \mathrm{~h}^{3} \mathrm{~L}^{3}+769 \mathrm{~h}^{4} \mathrm{~L}^{2}+402 \mathrm{~h}^{5} \mathrm{~L}\right\} \\
& G=\frac{1}{20160 h^{4}}\left\{4 L^{7}+70 h^{6}+490 h^{2} L^{5}+1750 h^{3} L^{4}+3346 h^{4} L^{3}+3220 h^{5} L^{2}+1200 h^{6} L\right\} \\
& H=\frac{1}{40320 h^{5}}\left\{L^{8}+16 h L^{7}+98 h^{2} L^{6}+280 h^{3} L^{5}+336 h^{4} L^{4}-309 h^{6} L^{2}-170 h^{7} L\right\} \\
& \text { with } \mathrm{L} \equiv\left(\mathrm{x}-\mathrm{x}_{\mathrm{n}+4}\right) \text {. }
\end{aligned}
$$

Equation (2.11) produces the coefficients of $\alpha_{j}(x)$ and $\beta_{j}(x)$ in equation (2.10) if

$$
t=\frac{x-x_{n+4}}{h}
$$

where $h=x_{n+j}-x_{n+j-1}$ Awoyemi ( 1999)

Evaluating the coefficients for $t=1$ yields

$$
y_{n+5}-3 y_{n+4}+3 y_{n+3}-y_{n+2}=\frac{h^{3}}{160}\left\{f_{n+5}+75 f_{n+4}+90 f_{n+3}-10 f_{n+2}+5 f_{n+1}-f_{n}\right\}---
$$

which is our new method with a difference scheme of order 5. A Taylor series expansion of (2.13) is taken as in Udo et al (2007) and like terms in powers of $h$ are collected to give

$$
T_{n+k}=C_{o}+C_{1} h+C_{2} h^{2}+\cdots+C_{p} h^{p}+C_{p+1} h^{p+1}
$$

According to definition 1 of Udo et al (2007)

$$
C_{0}=C_{1}=C_{2}=C_{3}=\ldots=C_{5}=0 \neq C_{p+1}=\frac{1}{240} ;------------(2 .
$$

where $C_{i}, i=0(1) p+1$, represents the sum of the coefficients of the like terms, $p$ the order of our method and $\mathrm{C}_{\mathrm{p}+1}$ is the first non zero term in our method.

The first and second characteristic polynomials of (2.13) are

$$
\rho(r)=r^{5}-3 r^{4}+3 r^{3}-r^{2} \text { and } \sigma(r)=\frac{1}{160}\left(r^{5}+75 r^{4}+90 r^{3}-10 r^{2}+5 r-1\right)
$$

have that

$\rho(1)=\rho^{\prime}(1)=\rho^{\prime \prime}(1)=0$ and $\rho^{m}(1)=3, \sigma(1)=6$. Applying definition 2 of Udo et al (2007) we see that $(2.13)$ is consistent.

If $\rho(r)={ }_{-}^{5}-3 r^{4}+3 r^{3}-r^{2}=0$, then $r=0,0,1,1,1$ 
What this means according to Fatunla (1988), is that (2.13) is a convergent method. According to Lambert (1973) and Fatunla (1988), the region of absolute stability can be established using

$$
h(r)=\frac{\rho(r)}{\sigma(r)}=\frac{160\left(r^{5}-3 r^{4}+3 r^{3}-r^{2}\right)}{\left(r^{3}+75 r^{4}+90 r^{3}-10 r^{2}+5 r-1\right)}
$$

Substituting $r=e^{i \theta}=\cos ^{\theta}+i \sin ^{\theta}$ into (2.16) gives

$$
h(\theta)=\frac{(160 \cos 5 \theta-3 \cos 4 \theta+3 \cos 3 \theta-\cos 2 \theta)+}{\frac{i(160 \sin 5 \theta-3 \sin 4 \theta+3 \sin 3 \theta-\sin \theta)}{(\cos 5 \theta+75 \cos 4 \theta+90 \cos 3 \theta-10 \cos 3 \theta+5 \cos \theta-1)+}}
$$

Rationalizing and simplifying (2.17) yields a system of the form

$$
h(\theta)=x(\theta)+i y(\theta) \text {.. }
$$

According to Fatunla (1988) and Awoyemi et al (2006), we ignore the imaginary part and evaluate $x(\theta)$ in (2.18) for $0 \leq \theta \leq 180$ to obtain $[0.3,20.4]$ as the region of stability of $(2.13)$.

Equation (1.1) suggests that we will need the first and second derivatives of (2.13), which are given as

$$
\begin{aligned}
& y_{n+5}^{\prime}=\frac{1}{2 h}\left(5 y_{n+4}-8 y_{n+3}+3 y_{n+2}\right)+\frac{h^{2}}{40320}\left(\begin{array}{l}
2664 f_{n+5}+27272 f_{n+4}+29808 f_{n+3}+1152 f_{n+2} \\
+1032 f_{n+1}-26 f_{n}
\end{array}\right) \\
& \text { and } \\
& y_{n+5}^{\prime \prime}=\frac{1}{h^{2}}\left(y_{n+4}-2 y_{n+3}+y_{n+2}\right)+\frac{h}{40320}\left(\begin{array}{c}
12682 f_{n+5}+12078 f_{n+4}+6756 f_{n+3} \\
-7244 f_{n+2}-1858 f_{n+1}+198 f_{n}
\end{array}\right) \quad---(2.20)
\end{aligned}
$$

respectivey.

We present below a summary of the properties of method (2.13) and those of its first and second derivatives. The analysis of the properties of methods (2.19) and (2.20) has been confirmed and are omitted here because of space. It follows the same process as that of (2.13).

Table 2.1: Summary of properties of method (2.13)

\begin{tabular}{|l|l|l|l|l|l|}
\hline Method & Order & $\mathrm{C}_{6}$ & Consistency & Zero stability & Convergent \\
\hline Main method (2.13) & 5 & 0.0042 & Yes & Yes & Yes \\
\hline First derivative (2.19) & 5 & -0.0076 & Yes & Yes & Yes \\
\hline $\begin{array}{l}\text { Second derivative } \\
(2.20)\end{array}$ & 5 & 0.0083 & Yes & Yes & Yes \\
\hline
\end{tabular}

\section{THE PREDICTOR}

In developing the predictor, we employ the same collocation procedure adopted for the main method (2.13) which yields the method of the form (2.10). Again putting $k=5$ and $t=\left(x-x_{n+4}\right) / h, i n(2.10)$ the coefficients $\alpha_{j}^{*}(x)$ and $\beta_{j}^{*}(x)$ can be determined.

Evaluating these coefficients at $\mathrm{t}=1$ gives our predictor for $y_{n+5}$ as

$$
y_{n+5}-3 y_{n+4}+3 y_{n+3}-y_{n+2}=\frac{h^{3}}{2}\left(f_{n+4}+f_{n+3}\right)
$$

The first and second derivations of (3.1) are 


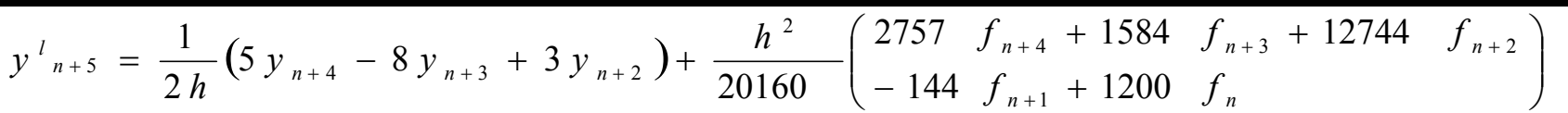

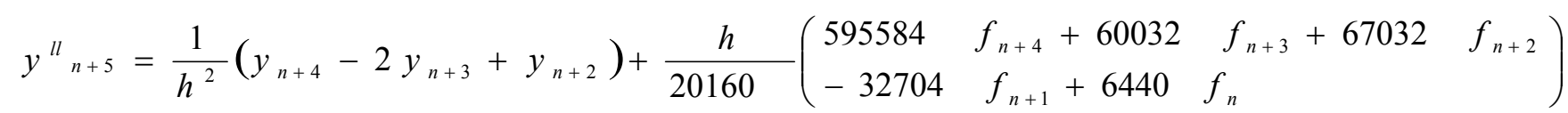

$$
\begin{aligned}
& ---(3.3)
\end{aligned}
$$

The predictors for $y_{n+4}$ and $y_{n+3}$, their respective first and second derivatives which are similarly derived are here listed

$$
y_{n+4}-3 y_{n+2}+3 y_{n+1}-y_{n}=\frac{h^{3}}{2}\left(f_{n+3}+f_{n+2}\right) \ldots
$$

having

$y_{n+4}^{1}=\frac{1}{2 h}\left(5 y_{n+3}-8 y_{n+2}+3 y_{n+4}\right)+\frac{h^{2}}{720}\left(894 f_{n+3}+228 f_{n+2}+198 f_{n+1}-48 f_{n}\right)$.

and

$y_{n+4}^{l l}=\frac{1}{h^{2}}\left(y_{n+3}-2 y_{n+2}+y_{n+1}\right)+\frac{h}{720}\left(189 f_{n+3}-1224 f_{n+2}+1014 f_{n+1} 248 f_{n}\right)$

while

$y_{n+3}-3 y_{n+2}+3 y_{n+1}-y_{n}=\frac{h^{3}}{2}\left(f_{n+2}+f_{n+1}\right)$.

having

$y_{n+3}^{l}=\frac{1}{2 h}\left(5 y_{n+2}-8 y_{n+1}+3 y_{n}\right)+\frac{h^{2}}{240}\left(298 f_{n+2}+124 f_{n+1}+18 f_{n}\right)$

$U$ sing the sameapproach $y_{n+3}$ was established.

It is striking to note that the results in (3.1) to (3.8) are identical to those derived in Awoyemi(2003).

Finally, we use Taylor series expansion to calculate the values of $y_{n+2}, y_{n+1}$ and their first and second derivatives at $x=x_{n}$ in (2.13),(2.16) and (2.17) see Awoyemi (2003). Thus

$$
\begin{gathered}
y_{n+i}=y\left(x_{n}+i h\right)=y\left(x_{n}\right)+i h y^{1}\left(x_{n}\right)+\frac{(i h)^{2}}{2 !} y^{i i}\left(x_{n}\right)+\frac{(i h)^{3}}{3 !} f_{n}+\frac{(i h)^{4}}{4 !} f_{n}^{1}+\ldots \\
\text { as } y_{n+i}^{1}=y^{1}\left(x_{n}\right)+(i h) y^{I I}\left(x_{n}\right)+\frac{(i h)^{2}}{2 !} f_{n}+\frac{(i h)^{3}}{3 !} f_{n}^{l}+\frac{(i h)^{4}}{4 !} f_{n}^{l l}+\ldots \ldots \ldots . . .
\end{gathered}
$$

$$
y_{n+i}^{I I}=y^{I I}\left(x_{n}\right)+(i h) f_{n}+\frac{(i h)^{2}}{2 !} f_{n}^{l}+\frac{(i h)^{3}}{3 !} f^{l l}+\ldots \ldots \ldots \ldots . . . .1=1,2
$$

where $f_{n}=f\left(x_{n}, y_{n}, y_{n}^{l}, y_{n}^{l l}\right), f_{n}^{(i)}=f^{(i)}\left(x_{n}, y_{n}, y_{n}^{l}, y_{n}^{l l}\right), \quad i=1,2$

Furthermore, we put (2.1) in the form

$f=f\left(x, y, y^{l}, y^{I I}\right)$

and find $f^{i}$ and $f^{I I}$ by partial derivative technique.

All predictors and their derivatives are analyzed and a summary is as presented

Table 3.1 Summary of properties of predictors

\begin{tabular}{|l|l|l|}
\hline Method & Order & $\mathrm{C}_{6}$ \\
\hline 3.1 & 5 & 0.0042 \\
\hline 3.2 & 5 & 0.0083 \\
\hline 3.3 & 5 & 0.0083 \\
\hline 3.4 & 5 & 0.157 \\
\hline 3.5 & 5 & -0.796 \\
\hline 3.6 & 5 & -0.328 \\
\hline 3.7 & 5 & -0.329 \\
\hline
\end{tabular}


Methods (3.1),(3.2),(3.3),(3.4),(3.5),(3.6) and (3.7) all methods were all found to be zero stable and consistent.

\section{NUMERICAL EXPERIMENT}

Three numerical examples on third order IVPs are considered to test the accuracy of the derived method.

\subsection{TEST PROBLEMS}

1. $y^{I I I}+4 y^{I}=x ; y(0)=0, y^{I}(0)=0, y^{I I}(0)=1,0 \leq x \leq 1$

Exact solution : $y(x)=\frac{3}{16}(1-\cos 2 x)+\frac{1}{8} x^{2}$

2. $y^{I I I}+y^{I}=0 ; y(0)=0, y^{I}(0)=1, y^{I I}(0)=2$

Exact Solution : $y(x)=2(1-\operatorname{Cos} x)+\operatorname{Sin} x$

3. $\quad y^{I I I}-\frac{1}{2} y y^{I I}=0 ; y(0)=y^{I}(0)=0, y^{I I}(0)=1$

The last problem is known as Blasius equation and has no analytical solution, Awoyemi et al (2006). The existence of solution is guaranteed by a theorem in Lambert (1973).

\subsection{NUMERICAL SOLUTION TO TEST PROBLEMS}

Here we present a manual framework for the solution of problem 1 using (2.13).A full manual solution is not only lengthy but tedious. The following starting values $y_{n}, y_{n}^{n}, y_{n}^{n}, y_{n+1}, y_{n+1}^{*}, y_{n+1}^{*}, y_{n+2}, y_{n+2}^{*}, y_{n+2}^{*}, y_{n+3} y_{n+3}^{*}, y_{n+3}^{*}, y_{n+4} y_{n+4}^{*}, y_{n+4}^{n}$, are needed for the evaluation of (2.13). The first three are our given initial conditions in (4.1a). The rest are gotten by taking a Taylor series of their respective expansions as in equations (3.1) to (3.9c). Setting $n=0$ in (2.13) gives

$y_{5}-3 y_{4}+3 y_{3}-y_{2}=\frac{h^{9}}{160}\left\{f_{5}+75 f_{4}+90 f_{3}-10 f_{2}+5 f_{1}-f_{0}\right\}$

where $y_{i}^{*}=f_{i}$

Schemes similar to that of (4.2) can be generated by setting $n=1,2,3$, in (2.13). Consequently an iterative process with $h=0.1$ can be carried out.

The results of the above solved problems using a computer program are here presented. Tables 5.1, 5.2 and 5.3 displays the exact results (YEX), calculated results (YC) and the errors (ER) arising from their difference. We considered the solution at $h$ equals $0.1,0.05$ and 0.025 .

Table 5.1: Problem 1 for $h=0.1$

\begin{tabular}{|c|c|c|c|}
\hline$X$ & YEX & $\mathrm{YC}$ & ER \\
\hline 0.20 & $0.1980106362 \mathrm{D}-01$ & $0.1980070242 \mathrm{D}-01$ & $0.3612062437 D-06$ \\
\hline 0.40 & $0.768674920 \quad \mathrm{D}-01$ & $0.7686353006 \mathrm{D}-01$ & $0.3961941555 \mathrm{D}-05$ \\
\hline 0.06 & $0.1645579210 \mathrm{D}-00$ & $0.1645442666 \mathrm{D}+00$ & $0.1365446766 \mathrm{D}-04$ \\
\hline 0.80 & $0.2729749104 \mathrm{D}+00$ & $0.272445367 \quad \mathrm{D}+00$ & $0.3037377736 \mathrm{D}-04$ \\
\hline 1.00 & $0.3905275319 \mathrm{D}+00$ & $0.3904749427 \mathrm{D}+00$ & $0.5258915799 \mathrm{D}-04$ \\
\hline
\end{tabular}

Table 5.2: Problem 2 for $\mathrm{h}=0.1$

\begin{tabular}{|c|c|c|c|}
\hline$X$ & YEX & $\mathrm{YC}$ & ER \\
\hline 0.20 & $0.2385361751 \quad D+00$ & $0.238531178 \quad D+00$ & $0.5730263181 \mathrm{D}-07$ \\
\hline 0.40 & $0.5472963543 \mathrm{D}+00$ & $0.547295972 \quad D+00$ & $0.6571494273 \mathrm{D}-06$ \\
\hline 0.06 & $0.9139712436 \mathrm{D}+00$ & $0.9139689071 \mathrm{D}+00$ & $0.2334493845 \mathrm{D}-05$ \\
\hline 0.80 & $0.1323942672 \mathrm{D}+01$ & $0.1323937192 \mathrm{D}+00$ & $0.5479875340 \mathrm{D}-05$ \\
\hline 1.00 & $0.1760866373 \mathrm{D}+01$ & $0.1760856072 \mathrm{D}+01$ & $0.1031080846 \mathrm{D}-04$ \\
\hline
\end{tabular}


Table 5.3: Problem 3 for $h=0.1$

\begin{tabular}{|c|c|c|c|}
\hline $\bar{X}$ & YEX & YC & ER \\
\hline 0.20 & $-0.3933917555 \mathrm{D}+01$ & $-0.3933520523 \mathrm{D}+01$ & $0.37970321956 \mathrm{D}-03$ \\
\hline 0.40 & $-0.7030904881 D+01$ & $-0.7029714371 D+01$ & $0.1190510675 \quad D-02$ \\
\hline 0.06 & $-0.1243810072 \mathrm{D}+02$ & $-0.1243444223 D+02$ & 0.3656483288 \\
\hline 0.80 & $-0.2220445624 \mathrm{D}+02$ & $-0.221944610 \quad D+02$ & $0.10001014363 \mathrm{D}-01$ \\
\hline 1.00 & $-0.4003573856 \mathrm{D}+02$ & $-0.4001086367 D+02$ & $0.2487489491 \quad \mathrm{D}-01$ \\
\hline
\end{tabular}

Table 5.4 Problem 1 for $\mathrm{h}=0.1,0.05,0.025$ and 0.0125

\begin{tabular}{|l|l|l|l|l|}
\hline $\mathrm{X}$ & $\mathrm{H}=0.1 \mathrm{ERROR}$ & 0.05 ERROR & 0.025 ERROR & 0.0125 ERROR \\
\hline 0.20 & $0.3612062437 \mathrm{D}-06$ & $0.6401835712 \mathrm{D}-07$ & $0.9128686838 \mathrm{D} .08$ & $0.1211163090 \mathrm{D}-08$ \\
\hline 0.40 & $0.3961941555 \mathrm{D}-05$ & $0.5609937763 \mathrm{D}-06$ & $0.7414099987 \mathrm{D}-07$ & $0.9516846713 \mathrm{D}-08$ \\
\hline 0.60 & $0.1365446766 \mathrm{D}-04$ & $0.1627079533 \mathrm{D}-05$ & $0.2356580064 \mathrm{D}-06$ & $0.2990465800 \mathrm{D}-07$ \\
\hline 0.80 & $0.3037377736 \mathrm{D}-04$ & $0.3947597266 \mathrm{D}-05$ & $0.5023483853 \mathrm{D}-06$ & $0.6333284569 \mathrm{D}-07$ \\
\hline 1.00 & $0.5258915799 \mathrm{D}-04$ & $0.6698377190 \mathrm{D}-05$ & $0.8441629226 \mathrm{D}-06$ & $0.1059195132 \mathrm{D}-06$ \\
\hline
\end{tabular}

Table 5.5 Problem 2 for $\mathrm{h}=0.1,0.05,0.025$ and 0.0125

\begin{tabular}{|l|l|l|l|l|}
\hline $\mathrm{X}$ & $\mathrm{H}=0.1 \mathrm{ERROR}$ & 0.05 ERROR & 0.025 ERROR & 0.0125 ERROR \\
\hline 0.20 & $0.8850700747 \mathrm{D} .07$ & $0.1134372979 \mathrm{D}-07$ & $0.1527019677 \mathrm{D}-08$ & $0.2001054600 \mathrm{~d}-09$ \\
\hline 0.40 & $0.6921191573 \mathrm{D}-06$ & $0.9425472591 \mathrm{D}-07$ & $0.1235577907 \mathrm{D}-07$ & $0.1582804221 \mathrm{D}-08$ \\
\hline 0.60 & $0.2371834769 \mathrm{D}-05$ & $0.3146139697 \mathrm{D}-06$ & $0.4053142377 \mathrm{D}-07$ & $0.5143681370 \mathrm{D}-08$ \\
\hline 0.80 & $0.5518098773 \mathrm{D}-05$ & $0.7186047399 \mathrm{D}-06$ & $0.9166154213 \mathrm{D}-07$ & $0.1157331031 \mathrm{D}-07$ \\
\hline 1.00 & $0.1033839056 \mathrm{D}-04$ & $0.1529238701 \mathrm{D}-05$ & $0.1684398196 \mathrm{D}-06$ & $0.2119696663 \mathrm{D}-07$ \\
\hline
\end{tabular}

Table 5.6: Comparison with Awoyemi et al (2006) for $\mathrm{K}=4$ using problem 2 for $\mathrm{h}=0.1$

\begin{tabular}{|c|ll|ll|l|l|}
\hline$X$ & $\begin{array}{l}\text { YC for K = 4 } \\
\text { (Awoyemi et al 2006) }\end{array}$ & $\begin{array}{l}\text { YC for K= } \\
\text { method) }\end{array}$ & (new & YEX \\
\hline 0.20 & 0.1980061481 & $\mathrm{D}-01$ & 0.2385361178 & $\mathrm{D}+00$ & 0.2385361751 & $\mathrm{D}+00$ \\
\hline 0.40 & 0.7686342432 & $\mathrm{D}-01$ & 0.547295972 & $\mathrm{D}+00$ & 0.5472963543 & $\mathrm{D}+00$ \\
\hline 0.60 & 0.1645441594 & $\mathrm{D}+00$ & 0.9139689071 & $\mathrm{D}+00$ & 0.9139712436 & $\mathrm{D}+00$ \\
\hline 0.80 & 0.2729444450 & $\mathrm{D}+00$ & 0.1323937192 & $\mathrm{D}+00$ & 0.1323942672 & $\mathrm{D}+01$ \\
\hline 1.00 & $0.39047488110 \mathrm{D}+00$ & 0.1760856072 & $\mathrm{D}+01$ & 0.1760866373 & $\mathrm{D}+01$ \\
\hline
\end{tabular}

\section{CONCLUSION}

An implicit zero stable method for the solution of third order IVPs is developed. The order of the main method and those of its predictors are found to be the same a case highly recommended by Fatunla (1988). The accuracy of this method is encouraging judging from the small error values. Three different test problems were considered for the different sizes of $h$. It was found that as $\mathrm{h}$ decreases, the method recorded improved accuracy (tables 5.4 and 5.5).

In Awoyemi et al (2006), the same set of problems were considered. The performance of the step length $\mathrm{k}=5$ method over that of step length $\mathrm{k}=4$ was evidently displayed in table 5.6, as results from both methods are compared with the exact value. However, in terms of computer time the $k=4$ method has a slight advantage over that of $k=5$ because of the number of variables per iteration. Hence, the choice of which method to adopt depends on accuracy and time.

\section{REFERENCES}

Awoyemi, D. O., 1996. An Efficient Two-Step Numerical Integrator for General Second Order Ordinary Differential Equation. ABACUS, Journal of Mathematical Association Of Nigeria, 24(2): 31-43.

Awoyemi, D. O., 1999. A Class Of Continuous Methods for General Second Order Initial Value Problems in Ordinary Differential Equation. Intern. J. Comp Math, 72, 29-37. 
Awoyemi, D. O., 2001. A New Sixth Order Algorithm for General Second Order Differential Equation. Intern. J. Comp Math, 77,117-124.

Awoyemi; D. O., 2003. A P Stable Linear Multi Step Method for Solving General Third Order Ordinary Differential Equation. Intern J. Comp Math, 50, Pp 987-993.

Awoyemi, D. O., Udo, M. O. and Adesanya, A. O., 2006. Non-Symmetric Collocation Method for Direct Solution of General Third Order Initial Value Problems of Ordinary Differential Equations. Journal of Nat. and Applied Sciences, 7 (1): Pp 31-37.

Bun, R. A., and Vasil'Yer, Y. D., 1992. A Numerical Method for Solving Differential Equations of any Orders. Comp. Math. Phys, 32, Pp 317-330.

Enright, W. H., 1974. Second Derivative Multi-Step Methods for Stiff ODEs. Siam Journal on Numerical Analysis 11, 321-331.

Fatunla, S. O., 1988. Numerical Methods for Initial Value Problems in Ordinary Differential Equations. Academic Press Inc., New York.

Lambert, J. D., 1973. Computational Methods In Ordinary Differential Equations. John Wiley, New York.

Twizell, E. H. and Khaliq, A. Q. M., 1984. Multiderivative Methods for Periodic IVPs. J. Numer. Anal, 21, 518-527.

Udo, M. O., Olayi, G. A. and Ademiluyi, R. A., 2007. Linear Multi-Step Method for Solution Of Second Order Initial Value Problems of Ordinary Differential Equations: A Truncation

Error Approach". Global Journ. of Mathematical Sciences.6 (2): Pp119-126. 